\title{
Genetics of bovine respiratory disease in cattle: can breeding programs reduce the problem?
}

\author{
Donagh P. Berry \\ Animal and Bioscience Research Department, Animal and Grassland Research and Innovation \\ Centre, Teagasc, Moorepark, Fermoy, Co. Cork, Ireland
}

Received 13 June 2014; Accepted 17 September 2014; First published online 1 December 2014

\begin{abstract}
Genetics is responsible for approximately half the observed change in performance internationally in well-structured cattle breeding programs. Almost all, if not all, individual characteristics, including animal health, have a genetic basis. Once genetic variation exists then breeding for improvement is possible. Although the heritability of most health traits is low to moderate, considerable exploitable genetic variation does exist. From the limited studies undertaken, and mostly from limited datasets, the direct heritability of susceptibility to BRD varied from 0.07 to 0.22 and the maternal heritability (where estimated) varied from 0.05 to 0.07 . Nonetheless, considerable genetic variation clearly exists; the genetic standard deviation for the direct component (binary trait), although differing across populations, varied from 0.08 to 0.20 while the genetic standard deviation for the maternal component varied from 0.04 to 0.07. Little is known about the genetic correlation between genetic predisposition to BRD and animal performance; the estimation of these correlations should be prioritized. (Long-term) Breeding strategies to reduce the incidence of BRD in cattle should be incorporated into national BRD eradication or control strategies.
\end{abstract}

Keywords: bovine respiratory disease, genetics, breeding, genetic improvement.

\section{Introduction}

Genetics is responsible for approximately half the observed change in performance internationally in well-structured cattle breeding programs. Performance change here implies improvement (e.g. milk yield in dairy cows and growth rate in beef) but also deterioration (e.g. reproductive performance in dairy and beef cattle). The advantage of genetic selection is that it is cumulative and permanent; although most well-structured breeding programs achieve genetic gain of $\sim 1 \%$ per annum, this equates to an annual $10 \%$ improvement by year 10 , or cumulatively a $55 \%$ improvement over those 10 years. Therefore, although management has a considerable influence on the performance levels of an individual, genetics, and therefore genomics, should also be part of a (long-term) strategy for improvement. Genetics creates the potential for performance, management realizes that potential, but disease destroys the potential.

\section{Technical terms}

Many technical terms are used in animal breeding which are briefly described (Berry et al., 2011):

Phenotype: The phenotype is simply the observed performance of an animal 'in the field' (e.g. the presence or absence of infection as measured by a positive or negative diagnostic test result).

Genotype: The definition of genotype varies. Animal breeders commonly use genotype to describe a particular strain of animals (e.g. animals of a given breed from a particular origin). Molecular geneticists, however, commonly use genotype to describe the genetic variants (i.e. alleles) an individual possesses at a particular position in its DNA, also known as a locus.

Genetic variation: A measure of the variation or differences within a population that is due to the differences in genetic merit of the animals. More commonly, genetic variation is expressed as the genetic standard deviation (i.e. the square root of the genetic variance) within a population.

Heritability: Heritability summarizes the proportion of phenotypic variation, or differences among a cohort of animals, attributable to genetic variation between individuals. Animal breeders commonly cite the narrow sense heritability $\left(h^{2}\right)$, 
which is the proportion of phenotypic variation attributed to additive genetic variation (i.e. allelic effects passed on from one generation to the next). Heritability varies from 0 (not heritable) to 1 (fully heritable). If the heritability is high, we can expect a large proportion of the phenotypic differences of the parents to be passed on to the progeny. Also, the larger the heritability, the greater the expressed phenotype resembles the underlying genetic merit of the animal. Low heritability does not necessarily imply slow/no genetic progress, and conversely, high heritability does not necessarily imply rapid genetic progress.

Maternal heritability: Maternal heritability is the proportion of phenotypic variation among offspring that is due to the genes expressed by the dam. A maternal heritability of calving difficulty is often quoted which encompasses, for example, the pelvic size of the dam. The direct heritability for calving difficulty is the effect of the alleles of the dam (and sire) on, for example, the size of the calf. Maternal heritability estimates have also been reported for weaning weight which includes genetic characteristics of the dam such as her milk yield.

Genetic correlation: A genetic correlation describes the strength of the linear relationship between two traits due to the genetic influences on each trait. It varies from -1 (strong negative relationship) to 0 (no relationship) to +1 (strong positive relationship between two variables). Genetic correlations can be due to the same genomic mutation affecting both traits (termed pleiotropic effect) or different genomic mutations affecting both traits but tending to, on average, be inherited together (i.e. linked).

Estimated breeding value (EBV): EBV is an estimate of the genetic merit for an animal for a given trait or series of traits based on an evaluation of all available data on the performance of an animal and close relatives. Using traditional methods of genetic evaluation, the true breeding value (or true genetic merit) is not known. The estimates of genetic merit are generally presented as the predicted transmitting ability (PTA) in dairy cattle or expected progeny difference (EPD) in beef cattle which is the EBV divided by two (i.e. an animal only passes half its genes to its progeny).

\section{Genetic improvement}

Annual genetic gain for a given trait may be described by the following equation (Rendel and Robertson, 1950):

$$
\Delta G=\frac{i \cdot r \cdot \sigma_{\mathrm{g}}}{\mathrm{L}}
$$

where $\Delta G$ is annual genetic gain; $i$ is the intensity of selection; $r$ is the accuracy with which you know the genetic merit of each animal; $\sigma$ is the genetic standard deviation (i.e. the square root of the genetic variance or simply just a measure of the genetic differences among animals); and $L$ is the generation interval. All else being equal, the greater the selection intensity (in other words, the smaller the proportion of extreme animals you select for a given trait), the greater will be the genetic gain for that trait. The accuracy of selection is affected by both the heritability of the trait and the information available on either the animal itself or its relatives. Figure 1 illustrates how the accuracy of selection, ignoring pedigree contributions, increases as the number of half-sib progeny with records increases for different heritability estimates. For a given number of progeny, the accuracy will be greater for higher heritability traits. However, accuracies of near unity are still achievable even for low heritability traits if sufficient information is available. Therefore, with the appropriate breeding program (i.e. large paternal half-sib groups) and infrastructure for the collection and storage of data, genetic gain in low heritability traits is certainly achievable if genetic variation is present. The accuracy of selection for a given trait may also be augmented by indirect selection for a correlated trait (Fig. 1) or exploiting genomic information in the genetic evaluations. The genetic standard deviation is a measure of the variation present in the population and the generation interval is the average age of the parents when its progeny are born. The generation interval is approximately 6 years in dairy and beef cattle (Mc Parland et al., 2007) but is expected to reduce with the implementation of genomic selection.

\section{Lessons from the past}

Most Holstein dairy populations worldwide are, on average, subfertile. This subfertility materialized from the now known antagonistic genetic correlation that exists between reproductive performance and milk production and the employed dairy breeding programs that aggressively selected for increased milk production alone. At the time, the antagonistic genetic correlation between milk production and reproductive performance was not known so therefore the repercussions of the implemented breeding programs were not established. The lack of knowledge of this correlation was due simply to a paucity of data on reproductive performance; the lack of data also hindered the ability to monitor reproductive performance over time which would also have alerted the deterioration in reproductive performance.

Nonetheless, once the issue was detected and the presence of genetic variance in reproductive performance quantified, reproductive performance was included in most international breeding goals (Miglior et al., 2005). Figure 2 illustrates the genetic trend in reproductive performance and milk production in the Irish dairy population; such trends are mirrored in almost all other international Holstein-Friesian populations. Moreover, the genetic trends are also reflected in phenotypic (i.e. field) trends. Thus, reproductive performance in Irish dairy cows (and most international Holstein-Friesian cow populations) is now improving due primarily to genetic selection for improved reproductive performance. Despite the antagonistic genetic correlation between milk production and reproductive performance, genetic merit for milk production is also increasing, albeit at a slower rate, to that prior to the inclusion of reproductive performance in the breeding goal.

The impact of current breeding goals in dairy and beef cattle on susceptibility to bovine respiratory disease (BRD) is not conclusively known. No large national datasets also exist for incidence of BRD with associated risk factors to evaluate whether any observed annual trends can be accredited to the prevailing 


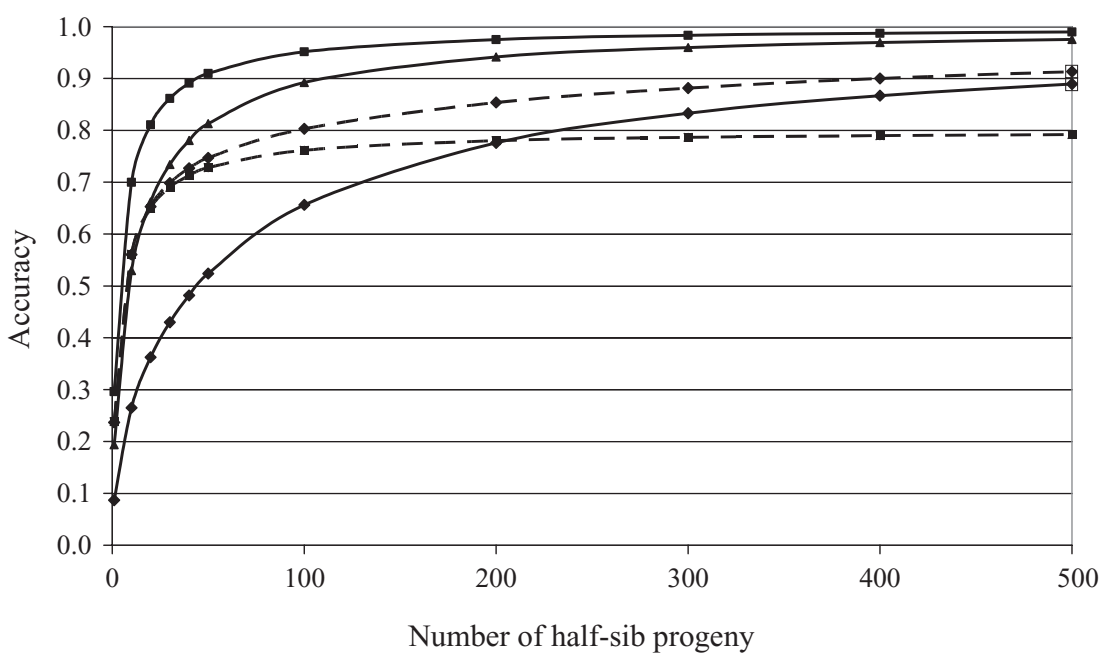

Fig. 1. Accuracy of selection across different numbers of half-sib progeny based on direct selection where the heritability of the trait is 0.03 (diamond with continuous line), 0.15 (triangle and continuous line), 0.35 (square with continuous line), and indirect selection where the goal trait is the 0.03 heritability trait and data are available on the 0.35 heritability trait alone (square with broken line) or also available on the 0.03 heritability trait (diamond with broken line), assuming a genetic correlation of 0.80 between both traits.

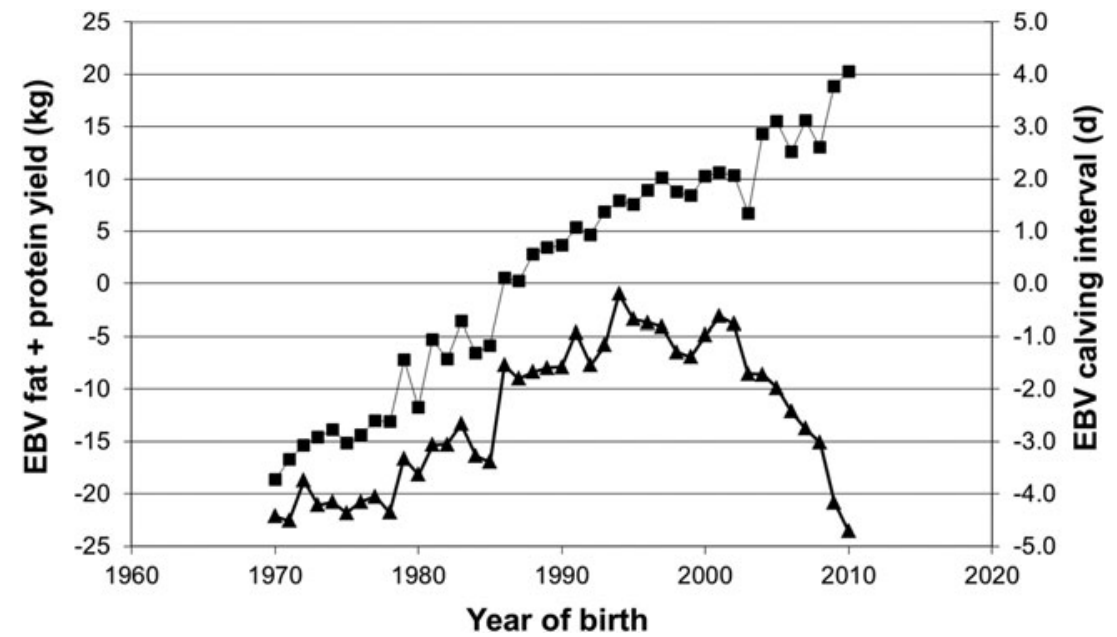

Fig. 2. Genetic trend for fat plus protein yield ( $\mathbf{\square})$ and calving interval ( $\boldsymbol{\Delta}$ ) for Irish Holstein-Friesian dairy cows. (Source: http://www.icbf.com)

breeding programs. Large scale collection of individual animal incidence of BRD and associated environmental risk factors is however probably prohibitive. Nonetheless, a lower cost option exists to evaluate the impact of current breeding strategies on genetic predisposition to BRD. A controlled experiment where animals genetically divergent for the breeding goal can be generated and the animals challenged with the pathogen. Results will indicate whether or not the genetically elite animals succumb more easily to the disease which will permit inferences to be made on the impact of the prevailing breeding strategies.

\section{Genetics of animal health}

Figure 3 summarizes the mean and variation in heritability estimates for a range of animal health and disease traits in both dairy and beef cattle. On average, the heritability of most health and disease traits is low and is consistent with the low heritability estimates of other viability and fitness traits like reproductive performance (Berry et al., 2014a, b). Nonetheless, variation in heritability estimates exists among populations. Heritability (as described earlier) is a function of the additive genetic variance present in the sample population as well as the corresponding non-(additive) genetic variation; both components are likely to differ between populations. Genetic variance is a function of the DNA allele frequency, the degree of allelic segregation, the allele substitution effect and the mode of allele action; for example no genetic variation exists among a population of cloned individuals. Genetic variation is therefore affected by evolutionary forces such as selection, migration, genetic drift, inbreeding and assortative mating. Genetic variation can also vary across time; for example, genetically susceptible animals may die or 


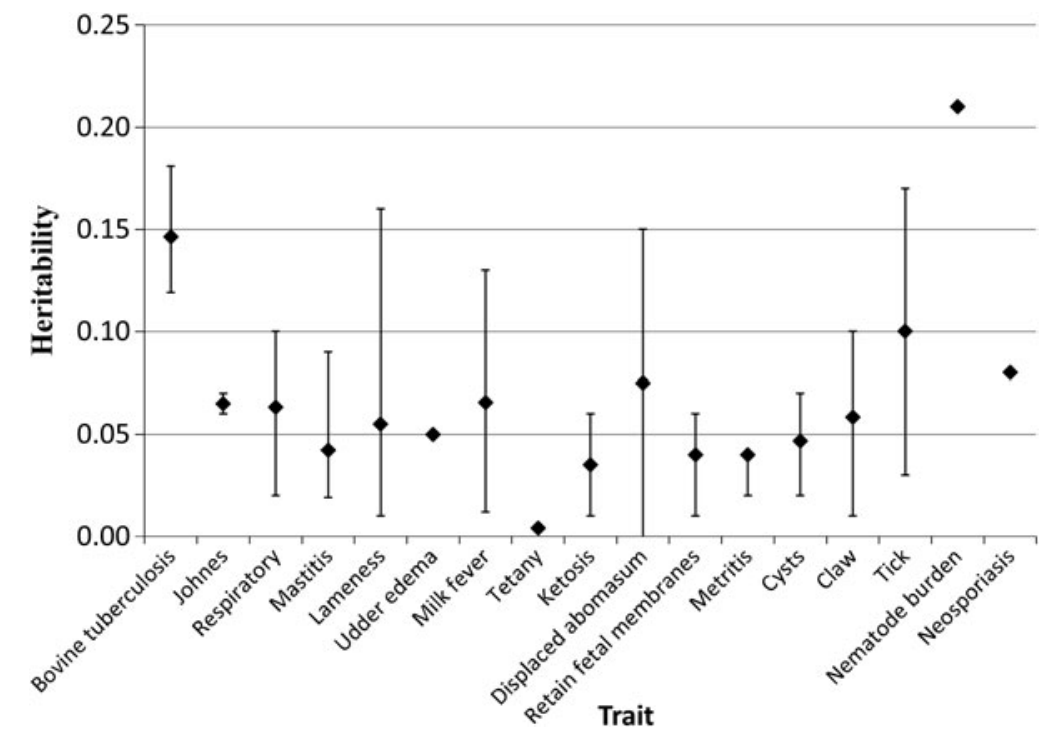

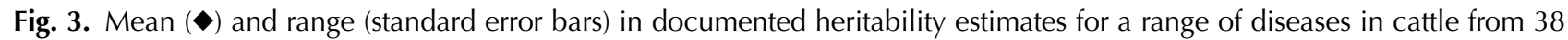
different populations.

be culled in the presence of a pathogen load and thus the genetic variation in the population of older animals may be less than in a population of younger animals. The estimated environmental variance can also vary across populations and will depend on, for example, factors such as the pathogen load present and even the complexity of the statistical model used in the analysis or the precision of the diagnostic test.

Although varying by health and disease trait, as well as population, there is a general tendency for an antagonistic genetic correlation to exist between animal health and performance (Berry et al., 2011). However, there is a paucity of large scale studies in beef cattle that is necessary for more precise estimates of the genetic correlations. This is not, however, to say that both traits cannot be improved simultaneously as evidenced by the genetic trends for both milk production and fertility in Fig. 2; both milk production and fertility are antagonistically correlated but once both are included in a breeding goal, the genetic merit for both attributes can improve simultaneously. This therefore implies that breeding goals that select for improved performance (which all breeding goals do) should take cognizance of health traits either directly, through the use of health phenotypes, or indirectly through the use of correlated traits such as general immunity traits, body condition score or survival (and possibly also fertility given it is expected to be linked to animal health).

\section{Genetics of BRD}

Few studies have attempted to quantify the genetics of susceptibility to BRD. Only four (relatively) large scale studies exist, including a study undertaken at the US Meat Animal research Center, Clay Center (Muggli-Cockett et al., 1992; Snowder et al., 2005), Iowa State University (Schneider et al., 2010), the national Norwegian dairy calf population (Heringstad et al., 2008) and the Irish national dairy and beef population (Berry et al., 2014a, b).
Schneider et al. (2010) reported a heritability for BRD of 0.11 $(0.06)$ in a population of pre-weaned US calves and 0.07 (0.04) in a population of feedlot cattle. However, of arguably more importance, especially in the genomics era, is the extent of the genetic variation present. The genetic standard deviation (transformed to a binary scale) for incidence of BRD in preweaned calves and feedlot cattle was 0.094 and 0.076 , respectively, signifying considerable exploitable genetic variation was present. Snowder et al. (2005) reported a direct heritability estimate of $0.22(0.01)$ and maternal heritability estimate of 0.07 (0.01) for BRD in pre-weaned beef calves at the US Meat Animal Research Center (MARC) from the years 1983 to 2000; the genetic standard deviation (transformed to a binary scale) was 0.13 and 0.07 for the direct and maternal component, respectively.

Heringstad et al. (2008), using data from $>250,000$ calves with a BRD incidence of $0.7 \%$, documented a heritability of 0.05 with a genetic standard deviation of 0.20 estimated using a threshold model. Norway is free from both infectious bovine herpersvirus-1 (BoHV-1/IBR) and bovine viral diarrhoea virus (BVDV) so therefore the most prevalent agents of BRD in this dataset were bovine respiratory syncytial virus (BRSV) and parinfluenza-3 virus (PI-3).

A compulsory national BVD eradication program was implemented in Ireland in 2012 (a voluntary scheme was implemented prior to 2012) for all cattle. An ear biopsy from all newborn calves is sent by each producer to a laboratory to quantify the presence of BVD virus. Therefore, for the analysis of BVD in Irish cattle almost 4 million calf BVD test results were available. The data were restricted to only calves born into a contemporary group of gestation, a maximum of 60 days apart, where at least one BVD positive calf was detected; this was undertaken to maximize, as far as possible, the equal likelihood of exposure of all animals to the virus. The final dataset consisted of 86,786 calves with an incidence of $9.75 \%$; this does not reflect the national average incidence as it was a highly edited 


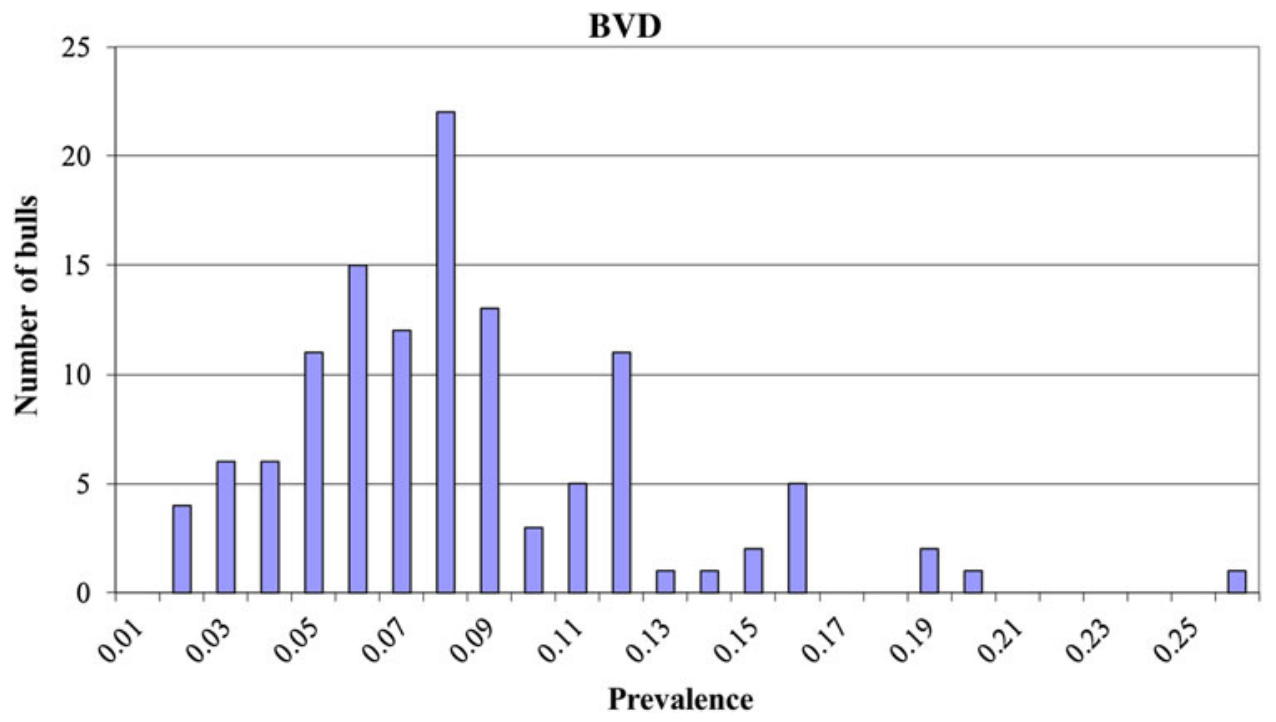

Fig. 4. Mean bvd prevalence per paternal half-sib group for dairy and beef sires with $>50$ progeny in $>10$ herds.

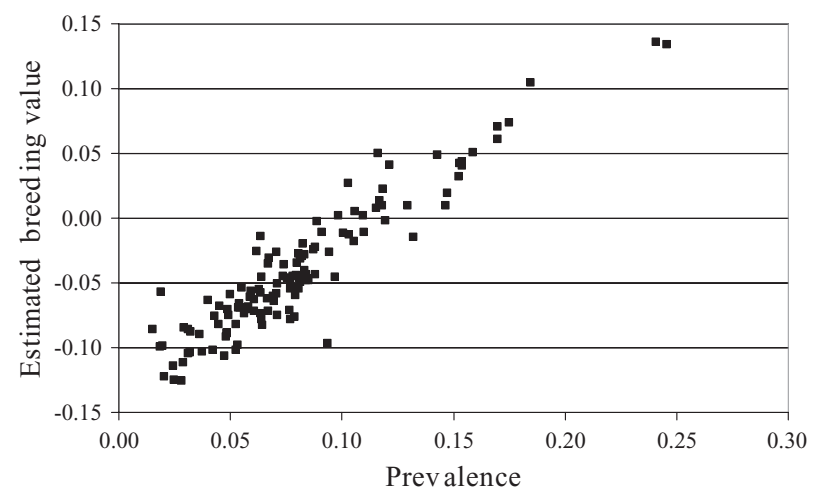

Fig. 5. Association between sire EBV for the direct genetic effect of BVD and sire mean progeny prevalence of BVD for dairy and beef sires with $>50$ progeny in $>10$ herds.

dataset. Primiparae and multiparae were considered separately (as they are managed separately on farm) and the incidence was slightly greater in primiparae.

The direct heritability of BVD was $0.16(0.02)$ and the maternal heritability was 0.05 (0.02). The genetic standard deviation for the direct component was 0.08 and the genetic standard deviation for the maternal component was 0.04 , implying the presence of considerable genetic variation. The existence of genetic variation in the Irish dataset is exemplified in Fig. 4 which presents a histogram of the mean prevalence of BVD per paternal half-sib group for sires with $>50$ progeny in $>10$ herds. Considerable (exploitable) variation clearly exists. Figure 5 depicts the relationship between sire EBV for BVD and mean progeny prevalence for BVD. A clear relationship exists substantiating the potential of animal breeding programs to reduce the incidence of BVD/BRD.

Data were also available from Ireland on IBR. Following edits, 5589 IBR records from 4523 dairy animals (different ages) from 53 contemporary groups were available for analysis; all contemporary groups had at least 1 home-born animal positive for antibodies to bovine herpes virus (BoHV-1/IBR). Of the 5589 records, 2581 were IRBgB and 3230 were IRBgE; 236 animals had records for both IRBgB and IRBgE. The incidence of $\mathrm{IBRgB}$ and $\mathrm{IBRgE}$ positive records in the database was 28.5 and $35.8 \%$, respectively. Heritability estimates for IBRgE (0.03-0.06; the lower estimate was not different from zero) was lower than observed for IBRgB (0.28-0.34). The repeatability estimates for both varied from 0.42 to 0.69 . The genetic standard deviation for the binary trait of IBR status was 0.07 and 0.15 for IBRgE and IBRgB, respectively, indicating considerable genetic variation present in these study herds.

The potential gains achievable in genetic merit for susceptibility to BRD is very much dependent on the breeding program implemented (i.e. phenotyping strategy), the genetic parameters for BRD (which we now know, albeit from limited populations) and the genetic correlations between BRD and other traits in the breeding goal (currently none known). With a good breeding program (i.e. high accuracy of selection either through a wellstructured and large progeny test or through the exploitation of genomic information), a response of 0.22 genetic standard deviations annually is possible. The genetic standard deviation for BRD from the US and Irish data appear to be approximately 0.10 . This, however, assumes no antagonistic genetic relationship exists between BRD and the entire breed goal. The presence of an antagonistic genetic correlation (which is very likely to exist) will reduce the response to selection.

\section{Conclusions}

Exploitable genetic variation in susceptibility to $\mathrm{BRD}$ exists among cattle with documented heritability estimates ranging from 0.07 to 0.22 for the direct component and from 0.05 to 0.07 for the maternal component. Little is known of the impact of current breeding strategies on genetic predisposition to BRD. Including BRD in breeding strategies as a component of national strategies to reducing the financial implications of BRD 
seems sensible. Breeding strategies for a trait have traditionally been achieved by recording the trait (i.e. BRD) on large populations of animals; although phenotypic information is still required, the use of genomic information in national genetic evaluations is partly in reducing the requirement for such phenotypic information on large populations of animals. For the doubters who suggest that genetics is not responsible for animal health, and that breeding strategies to improve animal health will be futile or too long-term, one only has to read the popular press (and some scientific articles) from a decade ago where the same argument was made for reproductive performance. Recent breeding programs are now credited in most countries for the observed improvement in reproductive performance in dairy cows. The same can be achieved for animal health.

\section{Acknowledgments}

Funding from the Research Stimulus Fund (GenCOST) is gratefully acknowledged.

\section{References}

Berry DP, Bermingham ML, Good M and More SJ (2011). Genetics of animal health and disease in cattle. Irish Veterinary Journal 64: 5.
Berry DP, Graham D, McClure J, McCarthy J and Sayers R (2014a). Heritable genetic variation in susceptibility to bovine viral diarrhea in dairy and beef cattle. In: Proceedings of the Agricultural Research Forum, 10-11th March 2014, Tullamore, Ireland.

Berry DP, Wall E and Pryce JE (2014b). Genetics and genomic of reproductive performances in dairy and beef cattle. Animal 8: 105-121.

Heringstad B, Chang YM, Gianola D and Østerås O (2008). Genetic analysis of respiratory disease in Norwegian Red calves. Journal of Dairy Science 91: 367-370.

Mc Parland S, Kearney JF, Rath M and Berry DP (2007). Inbreeding trends and pedigree analysis of Irish dairy and beef cattle populations. Journal of Animal Science 85: 322-331.

Miglior F, Muir BL and Van Doormaal BJ (2005). Selection indices in Holstein cattle of various countries. Journal of Dairy Science 88: 1255-1263.

Muggli-Cockett NE, Cundiff LV and Gregory KE (1992). Genetic analysis of bovine respiratory disease in beef calves during the first year of life. Journal of Animal Science 70: 2013-2019.

Rendel J, Robertson A (1950). Estimation of genetic gain in milk yield by selection in a closed herd of dairy cattle. Journal of Genetics $\mathbf{5 0}$ : $1-8$.

Schneider MJ, Tait RG, Ruble MV, Busby WD and Reecy JM (2010). Evaluation of fixed sources of variation and estimation of genetic parameters for incidence of bovine respiratory disease in preweaned calves and feedlot cattle. Journal of Animal Science 88: $1220-1228$.

Snowder GD, Van Vleck LD, Cundiff LV and Bennett GL (2005). Influence of breed, heterozygosity, and disease incidence on estimates of variance components of respiratory disease in preweaned beef calves. Journal of Animal Science 83: 1247-1261. 